\title{
Colossal electroresistance and colossal magnetoresistive step in paramagnetic insulating phase of single crystalline bilayered manganite $\left(\mathrm{La}_{0.4} \mathrm{Pr}_{0.6}\right)_{1.2} \mathrm{Sr}_{1.8} \mathrm{Mn}_{2} \mathrm{O}_{7}$
}

\author{
Y. Yamato, ${ }^{1}$ M. Matsukawa, ${ }^{1, a)}$ Y. Murano, ${ }^{1}$ R. Suryanarayanan, ${ }^{2}$ S. Nimori, ${ }^{3}$ M. Apostu, ${ }^{4}$ \\ A. Revcolevschi, ${ }^{2}$ K. Koyama, ${ }^{5}$ and N. Kobayashi ${ }^{5}$ \\ ${ }_{1}^{1}$ Department of Materials Science and Engineering, Iwate University, Morioka 020-8551, Japan \\ ${ }^{2}$ Laboratoire de Physico-Chimie de L'Etat Solide, CNRS, UMR8182, Université Paris-Sud, \\ 91405 Orsay, France \\ ${ }^{3}$ National Institute for Materials Science, Tsukuba 305-0047, Japan \\ ${ }^{4}$ Department of Physical, Theoretical and Materials Chemistry, Faculty of Chemistry, Al. I. Cuza University, \\ Carol I, 700506 Iasi, Romania \\ ${ }^{5}$ Institute for Materials Research, Tohoku University, Sendai 980-8577, Japan
}

(Received 11 December 2008; accepted 15 February 2009; published online 6 March 2009)

\begin{abstract}
We report a significant decrease in the low-temperature resistance induced by the application of an electric current on the $a b$-plane in the paramagnetic insulating (PMI) state of $\left(\mathrm{La}_{0.4} \mathrm{Pr}_{0.6}\right)_{1.2} \mathrm{Sr}_{1.8} \mathrm{Mn}_{2} \mathrm{O}_{7}$. A colossal electroresistance effect attaining $-95 \%$ is observed at lower temperatures. A colossal magnetoresistive step appears near $5 \mathrm{~T}$ at low temperatures below $10 \mathrm{~K}$, accompanied by an ultrasharp width of the insulator-metal transition. Injection of higher currents to the crystal and magnetic field cooling process cause a disappearance of the steplike transition. These findings have a close relationship with the presence of the short-range charge-ordered clusters pinned within the PMI matrix of the crystal studied. (C) 2009 American Institute of Physics.
\end{abstract} [DOI: 10.1063/1.3095598]

Perovskite manganites show a great variety of fascinating properties such as colossal magnetoresistance (CMR) effect and charge-ordered ( $\mathrm{CO})$ insulating phase. ${ }^{1}$ The most interesting one is the existence of a phase-separated state, the coexistence of antiferromagnetic (AFM) CO insulating and ferromagnetic metal (FMM) regions. ${ }^{2}$ Several recent works on metamagnetic transitions in phase-separated manganites have revealed that ultrasharp steps in magnetization curves appear at low temperatures. ${ }^{3,4}$ Hardy et al. ${ }^{4}$ proposed that the magnetization step of manganites arises from a martensitelike transformation associated with lattice strain between competing FMM and $\mathrm{CO}$ phases but questions have been raised.

Recently, it has been reported that the resistance of $\mathrm{CO}$ manganites exhibits a significant change when a strong electric field or current is applied. ${ }^{5,6}$ The electric current-induced resistance change results in a switching from the $\mathrm{CO}$ insulating to ferromagnetic metallic state. This phenomenon is called a colossal electroresistance (CER) effect, which is analogous to the term of CMR effect. The effect of electric fields on the $\mathrm{CO}$ state of the manganites is an issue of considerable interest not only on the basis of physical phenomena but also from a viewpoint of technological applications. There have been extensive studies on the effect of an applied electric current/voltage on the resistance of cubic manganites causing a huge negative ER effect so far. ${ }^{5-8}$ However, they have paid little attention on a bilayered manganite system showing an enhanced CMR effect due to its two-dimensional double layers of $\mathrm{MnO}_{2}$ sheets.

In this letter, we report a significant decrease in the lowtemperature resistance induced by the application of an electric current on the $a b$-plane in the paramagnetic insulating (PMI) state of Pr-substituted $\left(\mathrm{La}_{0.4} \mathrm{Pr}_{0.6}\right)_{1.2} \mathrm{Sr}_{1.8} \mathrm{Mn}_{2} \mathrm{O}_{7}$.

\footnotetext{
${ }^{a)}$ Electronic mail: matsukawa@iwate-u.ac.jp.
}

In our previous work, a steplike lattice deformation associated with the ultrasharp PMI-FMM transition has been investigated for single crystalline bilayered manganite $\left(\mathrm{La}_{0.4} \mathrm{Pr}_{0.6}\right)_{1.2} \mathrm{Sr}_{1.8} \mathrm{Mn}_{2} \mathrm{O}_{7} .{ }^{9}$ Next, we try to investigate the effect of an electric current on the colossal magnetoresistive step in the PMI phase of the crystal.

For the Pr-substituted $\left(\mathrm{La}_{1-z}, \mathrm{Pr}_{z}\right)_{1.2} \mathrm{Sr}_{1.8} \mathrm{Mn}_{2} \mathrm{O}_{7} \quad(z=0.6)$ crystal, a spontaneous FMM phase (originally present with no Pr substitution) disappears at ground state but a fieldinduced PMI to FMM transformation is observed over a wide range of temperatures. ${ }^{10,11}$ A magnetic $(H, T)$ phase diagram, established from magnetic measurements, is separated into three regions labeled as PMI, FMM, and bistable states, as shown in Fig. 1 of Ref. 12. Single crystals were grown by the floating zone method using a mirror furnace. The calculated lattice parameters of the tetragonal crystal structure of the crystals used here were shown in a previous report. ${ }^{11}$ The dimensions of the $z=0.6$ sample are $3.5 \times 2 \mathrm{~mm}^{2}$ in the $a b$-plane and $1.7 \mathrm{~mm}$ along the $c$-axis. The temperature dependence of resistivity was measured by using a closed cycle refrigerator with a conventional four-probe method. In particular, the electrodes were prepared using a gold past, to make a passage of the current uniform. Measurements of magnetoresistance (MR) were done using a $15 \mathrm{~T}$ superconducting magnet at Institute for Materials Research, Tohoku University. The normal sweep rate was set to be $0.26 \mathrm{~T} / \mathrm{min}$. Once we measured the isothermal MR at selected temperatures, the sample was kept fixed at high temperature of $150 \mathrm{~K}$ for $2 \mathrm{~h}$ for demagnetization. Checking the stability of sample's temperature, we restart recording the MR data. In our field-cooled (FC) measurements, the sample was cooled from 150 down to $4 \mathrm{~K}$ under the application of a magnetic field, we then removed the applied field and finally collected the MR data. 

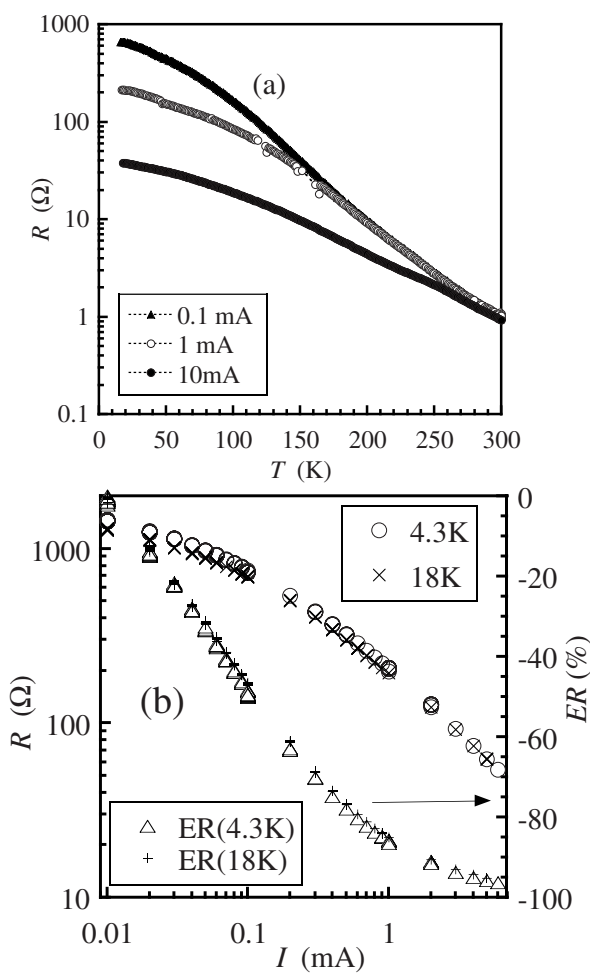

FIG. 1. (a) The temperature variation in $a b$-plane resistance of the $z=0.6$ crystal measured for various applied currents of $0.1,1$, and $10 \mathrm{~mA}$. (b) The ER and the ER ratio on the $a b$-plane at 4.3 and $18 \mathrm{~K}$ up to $6 \mathrm{~mA}$.

Let us show in Fig. 1 the temperature variation in the $a b$-plane resistance $R_{a b}(T)$ of the $z=0.6$ crystal measured for several applied currents of $0.1,1$, and $10 \mathrm{~mA}$. The substitution of Pr ion for La site suppresses the PMI-FMM transition temperature $T_{c}=\sim 120 \mathrm{~K}$ in the case of Pr-free crystal down to $T_{c}=\sim 90 \mathrm{~K}$ at $z=0.2$. For the present crystal with $z=0.6$, a spontaneous transition disappears even at the lowest temperature of $4 \mathrm{~K}$ and the system remains the PMI stable phase. At high current of $10 \mathrm{~mA}$, the temperature variation in $R_{a b}$ tends to saturate in the lower temperature region. Next, we examine the effect on an electric current on the resistance $R(I)$ of the $z=0.6$ crystal at the fixed temperatures of 4 and $18 \mathrm{~K}$. The current dependence of $R$ follows a strong nonlinearity and the magnitude of $R$ rapidly drops by more than one order of magnitude at higher currents. Here, an ER ratio is defined as $\left[R(I)-R\left(I_{\text {low }}\right)\right] / R\left(I_{\text {low }}\right) \times 100$, where $I_{\text {low }}$ is the lowest current value $(0.01 \mathrm{~mA})$. A CER effect reaching ER $=-95 \%$ at $I=5 \mathrm{~mA}$ is observed at lower temperatures. It is sure that a lower resistance state is achieved at higher currents but a current-induced insulator-metal (IM) transition observed in the $\mathrm{CO}$ cubic manganites is not noticed up to $10 \mathrm{~mA}$. We expect that the lower resistive state is realized by removing scattering centers of charge carriers due to the application of higher currents, such as the $\mathrm{CO}$ clusters being present within the PMI matrix. The formation of short-range charge ordering in the paramagnetic phase of an optimally doped parent crystal $\mathrm{La}_{1.2} \mathrm{Sr}_{1.8} \mathrm{Mn}_{2} \mathrm{O}_{7}$ has been observed in a previous study. ${ }^{13}$ Furthermore, neutron scattering measurements on a bilayered manganite $\mathrm{La}_{2-2 x} \mathrm{Sr}_{1+2 x} \mathrm{Mn}_{2} \mathrm{O}_{7}$ $(x=0.38)$ have revealed that the CE-type (zigzag FM chains with AFM interchain coupling) CO clusters freeze upon decreasing temperature from $T \sim 310 \mathrm{~K}$, preventing the formation of a long-range $\mathrm{CO}$ state. ${ }^{14}$ Here, we assume that the small CO clusters are pinned to the lattice through Jahn-
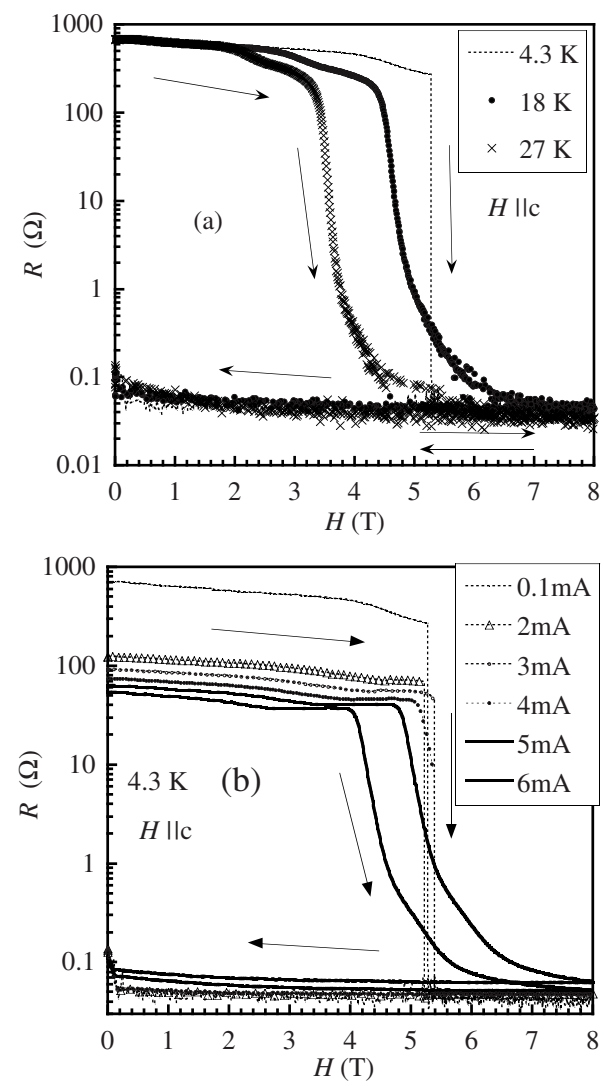

FIG. 2. (a) $a b$-plane MR data of the $z=0.6$ crystal at selected temperatures of $4.3,18$, and $27 \mathrm{~K}$ for $I=0.1 \mathrm{~mA}(H \| c)$. (b) $a b$-plane MR data as a function of an applied current from 0.1 up to $6 \mathrm{~mA}$.

Teller effect of $\mathrm{Mn}^{3+}$ ions as pointed out in Ref. 15. We then understand that they are depinned by the application of strong electric field, resulting in the nonlinear ER effect. Moreover, upon increasing $T$, a thermally activated depinning becomes more dominant, leading to a smaller ER effect. ${ }^{6}$ This pinning model qualitatively describes the CER effect enhanced at low $T$. As for the CER effect in the ferromagnetic insulating phase of $\mathrm{La}_{0.82} \mathrm{Ca}_{0.18} \mathrm{MnO}_{3}$, Mercone et $\mathrm{al}^{7}{ }^{7}$ argued that the electric current enhances transfer integral between $e_{g}$ orbitals of neighboring Mn sites, i.e., increases the $e_{g}$ bandwidth, which results in a resistance drop. To our knowledge, we have no experimental evidence for the enhanced bandwidth effect due to the application of a strong electric field on manganites.

Figure 2 shows MR data describing a magnetic-fieldinduced IM transition at selected temperatures of $4.3,18$, and $27 \mathrm{~K}(H \| c)$. The MR ratio $[R(H)-R(0)] / R(H) \times 100$, attains $\sim-10000 \%$ at $8 \mathrm{~T}$, exhibiting the typical CMR effect. A colossal magnetoresistive step suddenly appears with an ultrasharp width $(\sim 10 \mathrm{mT})$ of the IM transition when the applied field exceeds the critical field $\left(H_{c} \sim 5 \mathrm{~T}\right)$ at the lowest temperature below $10 \mathrm{~K}$. On the other hand, the higher temperature MR data follow a broad variation with the transition width $(\sim 1 \mathrm{~T})$. Now, we present in Fig. 2(b) the influence of electric current on the MR at $4.3 \mathrm{~K}$. First of all, the zero-field resistance $R(0)$ is reduced upon increasing the current passing the $z=0.6$ crystal as shown in Fig. 1(b). Next, at higher currents beyond $4 \mathrm{~mA}$, the magnetoresistive step completely disappears and instead we obtain a broad IM transition in MR similar to that realized at higher temperatures. Here, we give some comments on the problem of Joule heating. In our 

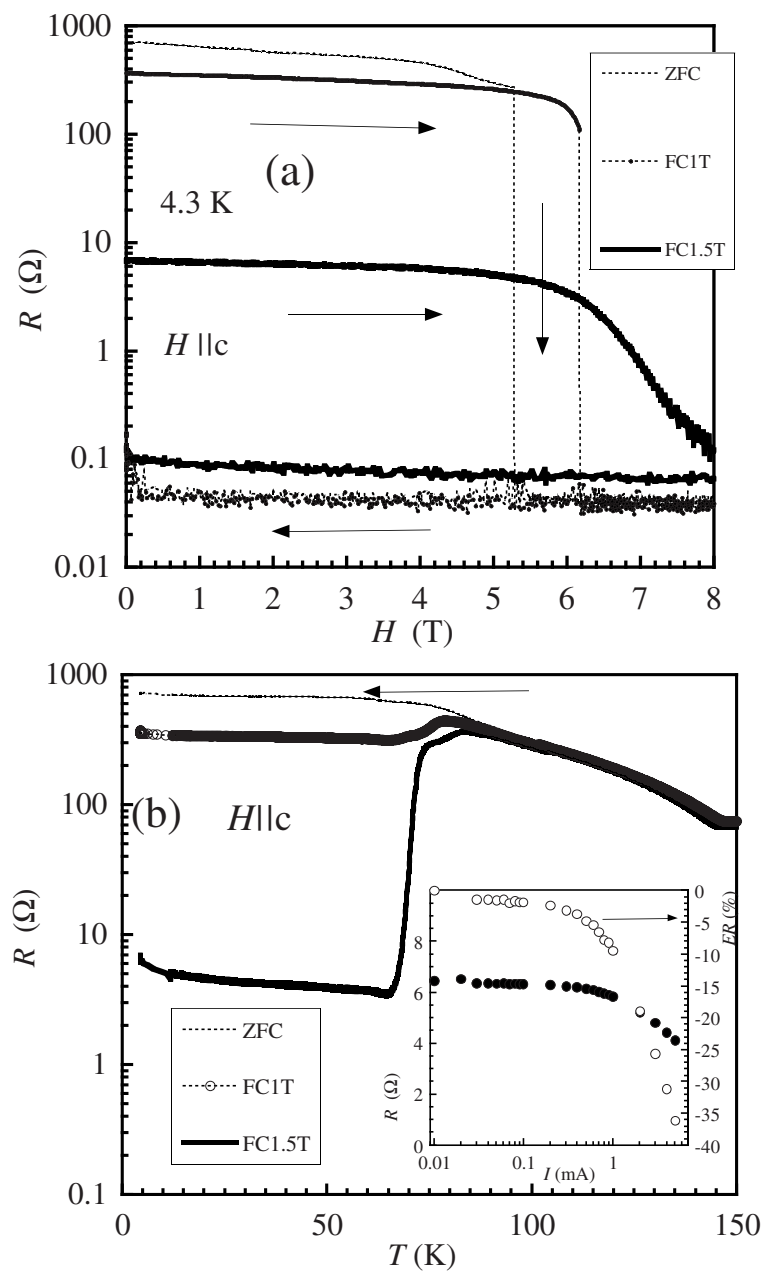

FIG. 3. (a) $a b$-plane MR data of the $z=0.6$ crystal after the field cooling runs, FC-1 T and FC-1.5 T, at $0.1 \mathrm{~mA}(H \| c)$. For comparison, the ZFC data are presented. (b) The temperature profile of the resistance monitored in the FC process from 150 down to $4 \mathrm{~K}$. The inset presents the ER and the ER ratio in the FC-1.5 $\mathrm{T}$ case at $4.3 \mathrm{~K}$.

previous study on the magnetostriction, we checked that low temperatures below $6 \mathrm{~K}$ are required for steplike transitions. If a temperature rise due to Joule heating exceeds the critical temperature $T_{\mathrm{sc}} \sim 6 \mathrm{~K}$, we expect the disappearance of the resistive step. In fact, our data up to lower currents of $I=3 \mathrm{~mA}$ exhibit a colossal resistive step, indicating that a temperature rise in the sample is limited below $T_{\mathrm{sc}}$. Moreover, the temperature dependence of $R$ shows a slight decrease even at intermediate temperatures above $50 \mathrm{~K}$. Accordingly, the Joule heating effect cannot explain the gigantic decrease in $R$ attaining $-93 \%$ at $I=3 \mathrm{~mA}$ before application of the magnetic field. We estimate a temperature rise $\delta T$ in the crystal due to Joule heating assuming a steadystate heat flow from the top of the sample toward its cold end under adiabatic condition (see Ref. 16). Joule heating at $5 \mathrm{~mA}$ gives $\delta T=1.8 \mathrm{~K}$ at the hot end within our rough approximation, resulting in $T>T_{\mathrm{sc}}$. Thus, it is difficult to judge whether the application of higher current or its associated heating dissipation causes the depressed step variation.

Finally, we examine the effect of field cooling on the MR of the $z=0.6$ crystal since the field cooling process increases a volume fraction of the FMM phase against the PMI matrix. The $a b$-plane MR data are presented in Fig. 3(a) after the sample experienced the FC mode (cooling fields of 1 and $1.5 \mathrm{~T})$. A low resistive state $(\sim 6 \Omega)$ is realized in the case of
FC-1.5T before the MR measurement, as shown in the temperature profile of $R$ of Fig. 3(b). The FC process causes a substantial suppression of the steplike transition from $H_{c}$ $\sim 5 \mathrm{~T}$ at ZFC to $6.2 \mathrm{~T}$ at FC-1T and for FC-1.5T, we then obtain the standard field-induced IM transition similar to that observed at higher $T$. The ER ratio is reduced to be $=-35 \%$ at $I=5 \mathrm{~mA}$ through FC-1.5T because the crystal is transformed to the low resistive state containing the large FMM region. This ER effect is probably attributed to a structural rearrangement of FMM clusters into filamentary shapes along the direction of an applied current. ${ }^{17}$

In summary, we have demonstrated the current-induced nonlinear decrease in the low-temperature resistance in the PMI phase of the $z=0.6$ crystal. The colossal magnetoresistive step at low temperature is suppressed due to injection of the higher currents to the crystal and through the field cooling process.

This work was supported by a Grant-in-Aid for Scientific Research from Japan Society of the Promotion of Science.

${ }^{1}$ Colossal Magnetoresistive Oxides, edited by Y. Tokura (Gordon and Breach, New York, 2000).

${ }^{2}$ Nanoscale Phase Separation and Colossal Magnetoresistance, edited by E. Dagotto (Springer, Berlin, 2003)

${ }^{3}$ R. Mahendiran, A. Maignan, S. Hebert, C. Martin, M. Hervieu, B. Raveau, J. F. Mitchell, and P. Schiffer, Phys. Rev. Lett. 89, 286602 (2002).

${ }^{4}$ V. Hardy, A. Maignan, S. Hebert, C. Yaicle, C. Martin, M. Hervieu, M. R. Lees, G. Rowlands, D. Mc K. Paul, and B. Raveau, Phys. Rev. B 68 , 220402(R) (2003)

${ }^{5}$ A. Asamitsu, Y. Tomioka, H. Kuwahara, and Y. Tokura, Nature (London) 388, 50 (1997).

${ }^{6}$ C. N. R. Rao, A. R. Raju, V. Ponnambalam, S. Parashar, and N. Kumar, Phys. Rev. B 61, 594 (2000).

${ }^{7}$ S. Mercone, A. Wahl, Ch. Simon, and C. Martin, Phys. Rev. B 65, 214428 (2002).

${ }^{8}$ H. Jain, A. K. Raychaudhuri, Ya. M. Mukovski, and D. Shulyatev, Appl. Phys. Lett. 89, 152116 (2006).

${ }^{9}$ M. Matsukawa, Y. Yamato, T. Kumagai, A. Tamura, R. Suryanarayanan, S. Nimori, M. Apostu, A. Revcolevschi, K. Koyama, and N. Kobayashi, Phys. Rev. Lett. 98, 267204 (2007).

${ }^{10}$ Y. Moritomo, Y. Maruyama, T. Akimoto, and A. Nakamura, Phys. Rev. B 56, R7057 (1997)

${ }^{11}$ M. Apostu, R. Suryanarayanan, A. Revcolevschi, H. Ogasawara, M Matsukawa, M. Yoshizawa, and N. Kobayashi, Phys. Rev. B 64, 012407 (2001).

${ }^{12}$ M. Matsukawa, K. Akasaka, H. Noto, R. Suryanarayanan, S. Nimori, M. Apostu, A. Revcolevschi, and N. Kobayashi, Phys. Rev. B 72, 064412 (2005).

${ }^{13}$ L. Vasiliu-Doloc, S. Rosenkranz, R. Osborn, S. K. Sinha, J. W. Lynn, J. Mesot, O. H. Seeck, G. Preosti, A. J. Fedro, and J. F. Mitchell, Phys. Rev. Lett. 83, 4393 (1999).

${ }^{14}$ D. N. Argyriou, J. W. Lynn, R. Osborn, B. Campbell, J. F. Mitchell, U. Ruett, H. N. Bordallo, A. Wildes, and C. D. Ling, Phys. Rev. Lett. 89, 036401 (2002).

${ }^{15}$ A. Guha, A. Ghosh, A. K. Raychaudhuri, S. Parashar, A. R. Raju, and C. N. R. Rao, Appl. Phys. Lett. 75, 3381 (1999).

${ }^{16} \mathrm{~A}$ steady-state heat flow is given by $Q=-\kappa S d T / d x(0 \leq x \leq L)[\kappa: c$-axis thermal conductivity, $d T / d x(\| c)$ : temperature gradient along the heat flux, and $S(\| a b)$ and $L(\| c)$ : the cross section and length of the sample]. Integrating it with respect to $x$, we then obtain $\delta T=I^{2} R L /(2 \kappa S)$ at the hot surface if the heat flux is proportional to the volume fraction of the sample in such a way as $Q(x)=I^{2} R(L-x) / L$. For $I=3$ and $5 \mathrm{~mA}$, we estimate $\delta T=\sim 1.0$ and $1.8 \mathrm{~K}$, respectively. We note $\kappa_{c}=1 \mathrm{~mW} / \mathrm{cm}$ at $4.3 \mathrm{~K}$ which is extrapolated from the data in a literature. M. Matsukawa, M Narita, T. Nishimura, M. Yoshizawa, M. Apostu, R. Suryanarayanan, A. Revcolevschi, K. Itoh, and N. Kobayashi, Phys. Rev. B 67, 104433 (2003)

${ }^{17}$ S. Dong, H. Zhu, and J. M. Liu, Phys. Rev. B 76, 132409 (2007). 